\title{
Spatial Coherence Based Beamforming in Multi-Line Transmit Echocardiography
}

\author{
Giulia Matrone \\ Dept. of Electrical, \\ Computer and Biomedical \\ Engineering \\ University of Pavia \\ Pavia, Italy \\ giulia.matrone@unipv.it
}

\author{
Alessandro Ramalli \\ Dept. of Cardiovascular \\ Sciences \\ KU Leuven \\ Leuven, Belgium
}

\author{
Jan D'hooge \\ Dept. of Cardiovascular \\ Sciences \\ KU Leuven \\ Leuven, Belgium
}

\author{
Piero Tortoli \\ Dept. of Information \\ Engineering \\ University of Florence \\ Florence, Italy
}

\author{
Giovanni Magenes \\ Dept. of Electrical, \\ Computer and Biomedical \\ Engineering \\ University of Pavia \\ Pavia, Italy
}

\begin{abstract}
Multi-line transmission (MLT) has recently gained interest for high frame-rate ultrasound imaging in cardiac applications, and several approaches have been proposed to address its main limitation, i.e. the limited contrast due to crosstalk artifacts. In this work, we analyze and compare the MLT imaging performance of four spatial-coherence-based beamformers, i.e. Filtered-Delay Multiply and Sum (F-DMAS), Phase Coherence (PC), Sign Coherence (SC) beamforming and Coherence Factor (CF) weighting. Simulations and experimental phantom acquisitions were carried out with the ULA-OP system using a $2 \mathrm{MHz}$ phased array to scan a $90^{\circ}$ imaging sector. Standard focused mode (i.e. Single Line Transmission, SLT) and MLT with 4 simultaneously transmitted beams were implemented. Results demonstrate that higher crosstalk suppression, improved lateral resolution and contrast are achieved by employing any of the analyzed coherence-based methods in MLT imaging as compared to Delay and Sum (DAS). Such beamformers can thus represent a promising solution for cardiac applications, achieving a similar or even better image quality than standard methods, but with higher frame-rate.
\end{abstract}

Keywords-beamforming, coherence factor, delay multiply and sum, high frame-rate imaging, multi-line transmission, phase coherence, ultrasound imaging

\section{INTRODUCTION}

High frame rate ultrasound imaging can be achieved by implementing different techniques, in transmission (TX) and/or reception ( $\mathrm{RX})$. In standard B-mode imaging, an image frame is acquired by sequentially transmitting and receiving a focused beam, until the entire region of interest has been covered line by line, while, in high frame-rate imaging, specific strategies reduce the number of TX events or parallelize the reconstruction of multiple image lines.

Multi-line transmission (MLT) consists in simultaneously transmitting multiple ultrasound beams in different directions, thus increasing the frame rate by a factor equal to the number

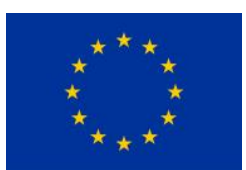

This work was partially supported by the University of Pavia under the Blue Sky Research project MULTIWAVE. A. Ramalli was supported by the European Union's Horizon 2020 research and innovation programme under the Marie SkłodowskaCurie grant agreement No 786027 (ACOUSTIC project). of simultaneous TX beams. This technique has recently gained interest in echocardiographic applications [1, 2], where achieving a higher frame-rate is of the uttermost importance, since the tracking of heart structures or the precise timing of rapid cardiac events could provide valuable diagnostic information. However, MLT suffers from a partial degradation of the image quality, due to crosstalk artifacts generated by interferences among the beams. To address this problem, Tukey apodization in TX and RX has been proposed in [3] as a method to reduce TX and RX crosstalk respectively, partially worsening lateral resolution. Other methods have also been proposed to this aim, such as miminum variance beamforming [4], adaptive apodization [5], harmonic imaging [6], and Filtered-Delay Multiply and Sum (F-DMAS) beamforming [7].

In this work, we analyze the MLT imaging performance of other beamforming techniques, namely Phase Coherence (PC) [8], Sign Coherence (SC) [8] beamforming and Coherence Factor (CF) weighting [9], that, similarly to F-DMAS, are based on the concept of spatial coherence of backscattered echoes. Simulations of the system's point spread function (PSF) and imaging experiments on a tissue-mimicking phantom using a phased array probe are carried out to compare these methods in MLT, showing their superior performance for potential use in echocardiography, as compared to simple Delay and Sum (DAS) with Tukey apodization.

\section{MATERIALS AND MethodS}

\section{A. Multi-Line Transmission}

In standard ultrasound B- mode imaging, a single focused beam is transmitted and received for every TX event (i.e. Single Line Transmission, SLT) and this process is repeated sequentially $N_{L I N E S}$-times, where $N_{\text {LINES }}$ is the number of image scan lines to be acquired.

The MLT imaging technique consists in acquiring an ultrasound image frame by simultaneously transmitting several $\left(N_{M L T}\right)$ beams and then beamforming the corresponding scan lines in parallel in RX. Hereby, the number of TX events is reduced by a factor $N_{M L T}$, resulting in a frame-rate improvement by $N_{M L T}$. To do so, each $n$-th transducer of the $N$ element active aperture is excited using a signal that is obtained by summing up the signals that would be required to focus the 
beam in each one of the desired TX directions, thus applying different focusing delay values. Then, the echoes received after each TX event are beamformed in parallel along the $N_{M L T}$ scan lines corresponding to the selected TX directions.

The frame-rate improvement, however, comes at the cost of a reduced image quality, due to interferences among the simultaneous beams, which in turn generate the so-called TX/RX crosstalk artifacts in the final image [3]. TX crosstalk is generated when the TX beam side lobes interfere with the RX beam main lobe, while RX crosstalk arises from interferences between the TX beam main lobe and RX beam side lobes.

\section{B. Coherence Factor weighting}

The CF is defined as the ratio between the coherent and incoherent sum of the dynamically delayed radiofrequency (RF) signals $\left(s_{n}(t)\right)$ [10]. As such, CF is expected to have a high value (approaching 1) when received signals are highly coherent, i.e. they come from the beam main-lobe, and lower values (down to 0 ) in the presence of strong contributions from off-axis signals, which instead represent interferences and noise. Thus, the CF represents a metrics to evaluate the quality of focusing and can be used to weight the DAS-beamformed signals $v_{\text {das }}(t)$ [9] to improve image resolution and contrast, as follows:

$$
v_{c f}(t)=v_{d a s}(t) \cdot C F(t)=v_{d a s}(t) \cdot \frac{\left|\sum_{n=1}^{N} s_{n}(t)\right|^{2}}{N \sum_{n=1}^{N}\left|s_{n}(t)\right|^{2}} .
$$

\section{Phase and Sign Coherence beamforming}

The PC algorithm [8] evaluates the dispersion of the received signals after focusing, in order to derive a metric that accounts for signal coherence. The PC factor (PCF), in fact, is defined as:

$$
P C F(t)=\max \left\{0,1-\frac{\gamma}{\sigma_{0}} \sigma\left(\varphi_{n}(t)\right)\right\}
$$

where $\sigma_{0}=\pi / \sqrt{3}$ is the standard deviation of a uniform distribution in $[-\pi ; \pi], \sigma\left(\varphi_{n}(t)\right)$ is the standard deviation of the signal instantaneous phases $\varphi_{n}(t)$, and $\gamma$ (in the range $[0 ; 1]$ ) corresponds to the sensitivity of PCF, where higher values increase the rejection of out-of-focus signals.

Sign coherence [8] instead considers the distribution of signs of received signals; the SC Factor (SCF) is given by:

$$
S C F(t)=\left|1-\sigma\left(b_{n}(t)\right)\right|^{p}
$$

where $b_{n}(t)=\operatorname{sign}\left(s_{n}(t)\right)$, and $p \geq 0$ is a user-defined parameter for SCF sensitivity tuning.

Both PCF and SCF are used to weight the DASbeamformed image as follows:

$$
\begin{gathered}
v_{p c f}(t)=v_{d a s}(t) \cdot P C F(t) \\
v_{s c f}(t)=v_{d a s}(t) \cdot S C F(t)
\end{gathered}
$$

\section{Filtered Delay Multiply and Sum Beamforming}

F-DMAS is a non-linear beamforming algorithm based on the computation of the spatial autocorrelation of the RX aperture [11]. Basically, it consists in focusing the RF signals received by the array, rescaling their amplitude by means of a signed square root operation, combinatorially coupling and multiplying them. The resulting signals from each multiplication stage are finally summed and band-pass filtered (BPfilter) at twice the frequency of the excitation pulse. The following equation resumes the F-DMAS beamformer steps:

$$
v_{\text {fdmas }}(t)=B \text { Pfilter }\left\{\sum_{n=1}^{N-1} \sum_{m=n+1}^{N} \operatorname{sign}\left(s_{n}(t) s_{m}(t)\right) \cdot \sqrt{\left|s_{n}(t) s_{m}(t)\right|}\right\}
$$

\section{E. Simulations and Experiments}

The performance of the previously described coherencebased beamformers and of DAS plus Tukey RX apodization (i.e. DAS-T) were evaluated and compared in MLT imaging simulations and phantom experiments.

Field II $[12,13]$ was employed to simulate the PSF, which was used to assess lateral resolution. A $2 \mathrm{MHz}, 64$-element phased-array probe, with a $0.34 \mathrm{~mm}$ pitch and $1.2 \mathrm{~mm}$ element height was modelled; the simulated image sector was $90^{\circ}$ wide. 192 scan lines were acquired, with a TX focal depth of $70 \mathrm{~mm}$. The excitation pulse consisted of a 2-cycle sinusoidal burst at $2 \mathrm{MHz}$ with Hanning weighting. A single pointscatterer was placed at $(x, z)=(0,70) \mathrm{mm}$.

In experiments, the ULA-OP scanner [14], coupled to an Esaote PA230 phased-array probe (pitch: $0.17 \mathrm{~mm}$ ), was employed by activating the 64 odd elements of the array, in order to achieve the same configuration used in simulations. The maximum TX signal amplitude was $25 \mathrm{Vpp}$. A CIRS tissue-mimicking phantom, model 040GSE was scanned and pre-beamforming RF signals, sampled at $50 \mathrm{MHz}$, were acquired by the system and then beamformed offline in Matlab.

Both a SLT scan and MLT with 4 TX beams (4MLT) were implemented. Tukey apodization (with $\alpha=0.5$ ) was always applied in TX to suppress TX crosstalk. The previously listed RX beamformers were then implemented and the following parameter values were empirically determined: for F-DMAS, the band-pass filter was set between 1.5-7 MHz; for PC, $\gamma=1$ was employed; $p=0.7$ was chosen for SC.

\section{RESULTS}

Fig. 1 and 2 show the simulated PSF image and its lateral profile, respectively, obtained with $4 \mathrm{MLT}$ and different RX beamforming methods. The lateral resolution (LR) was evaluated by measuring the main-lobe width at $-6 \mathrm{~dB}$, see Table I. As a reference, also the resolution values obtained in standard SLT are provided in Table I, while images only show the DAS-T SLT case. Fig. 1 clearly demonstrates that coherence-based beamformers generally achieve a better image quality than DAS-T, as the PSF main lobe is always narrower and side lobes are significantly reduced. PC, SC and CF provide a slightly better lateral resolution than F-DMAS, and the narrowest PSF is that obtained with PC. Besides, RX 


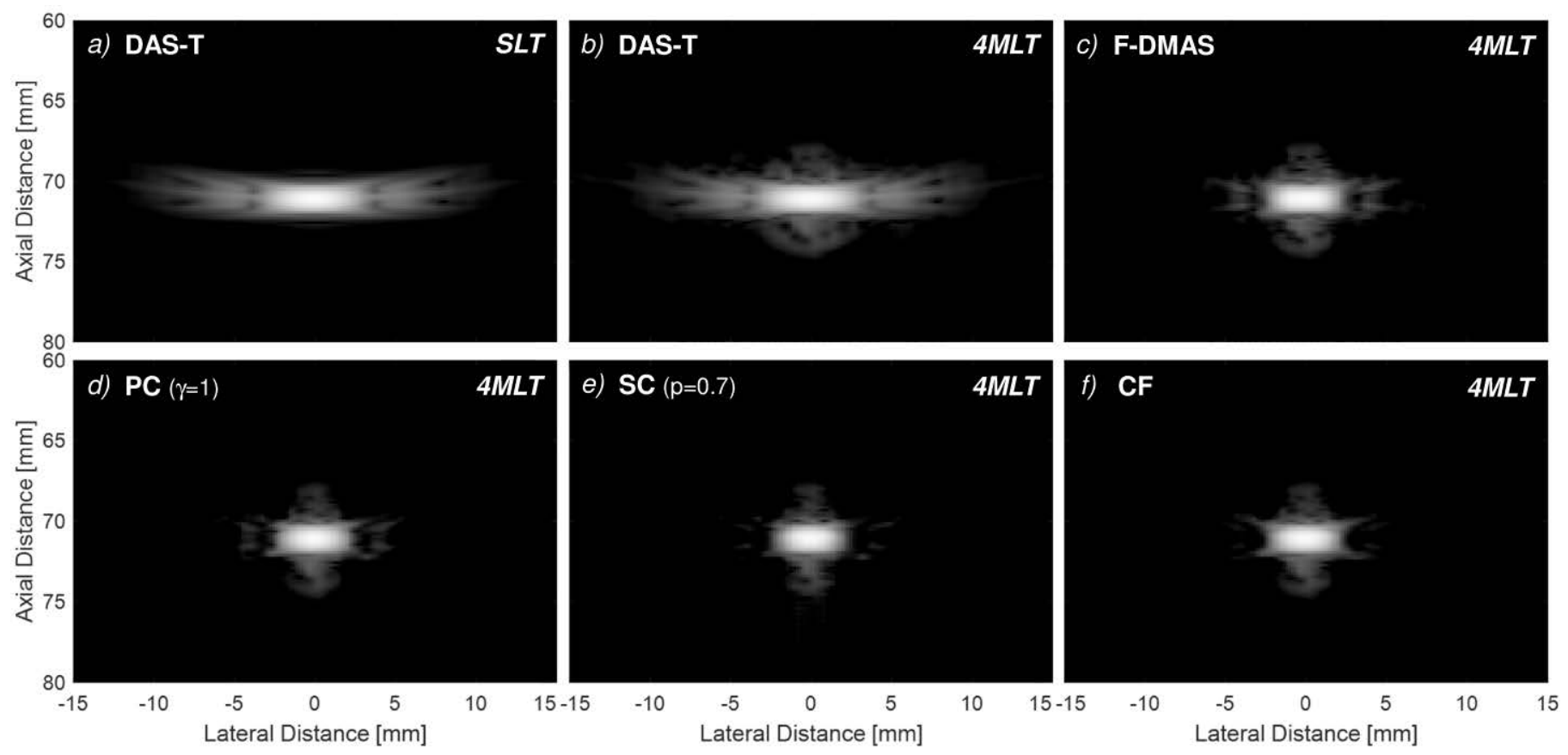

Fig. 1. Simulated PSF images obtained with Tukey TX apodization and: a), b) DAS beamforming with Tukey RX apodization (DAS-T), c) F-DMAS beamforming, d) PC with $\gamma=1$, SC with $p=0.7$, and CF weighting. Panel a) represents a standard SLT scan as a reference, while panels b)-f) are obtained by implementing 4MLT. Images are represented over a $70 \mathrm{~dB}$ dynamic range (log scale).

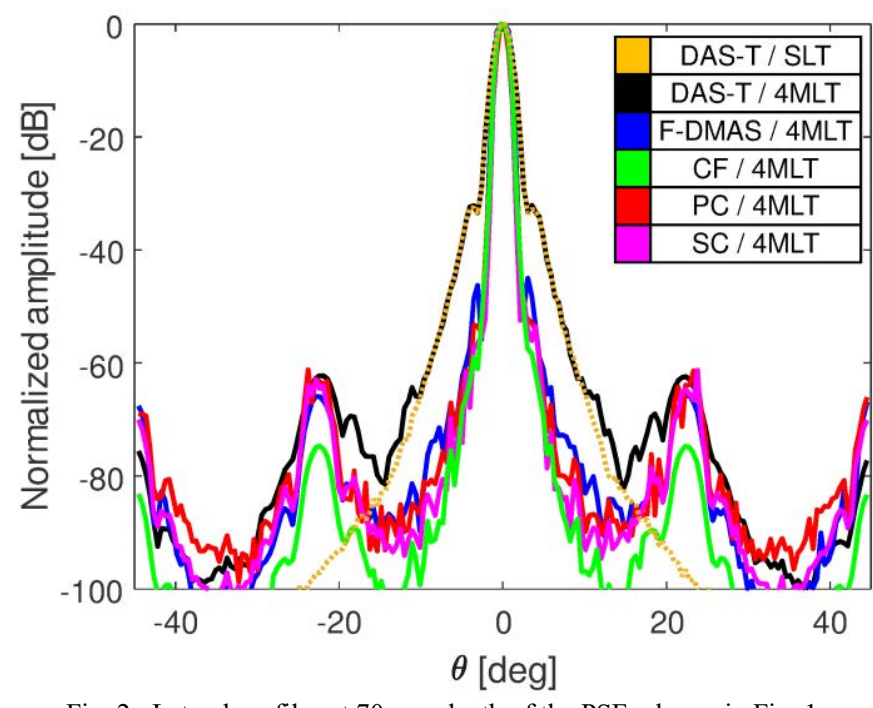

Fig. 2. Lateral profiles at $70 \mathrm{~mm}$ depth of the PSFs shown in Fig. 1.

crosstalk rejection is improved when applying CF weighting, as can be observed in Fig. 2 by looking at the peaks at the right and left sides of the PSF main lobe.

The phantom images in Fig. 3 demonstrate the superior performance of coherence-based beamformers in MLT also in terms of contrast. The contrast ratio $(\mathrm{CR})$ and contrast-to-noise ratio (CNR) were measured as in [7] on the anechoic cyst at 70 $\mathrm{mm}$ depth, using two circular areas (5 mm diameter) inside and outside the cyst (Fig. 3a). The same background area was also considered to compute the speckle signal-to-noise-ratio (sSNR) [7]. All measured values are reported in Table I. In general, coherence-based beamformers achieve a lower CNR and sSNR as compared to DAS: the speckle looks sharper, the mean background value decreases and its variance gets higher, causing a worse speckle uniformity and lowering these parameters, in particular when $\mathrm{CF}$ or $\mathrm{PC} / \mathrm{SC}$ are used. On the other hand, the most significant improvement is in terms of CR: with coherence-based methods the CR in SLT and 4MLT is at least $10 \mathrm{~dB}$ and $6 \mathrm{~dB}$ higher than with DAS and Tukey RX apodization, respectively. The highest values are achieved with $\mathrm{CF}$ weighting $(-38.6 \mathrm{~dB}$ in SLT and $-26.3 \mathrm{~dB}$ in 4MLT vs. $-22.3 \mathrm{~dB}$ and $-15 \mathrm{~dB}$ for DAS), thanks to the strong $\mathrm{RX}$ crosstalk reduction and narrow beam main-lobe, that improve both contrast and lateral resolution.

\section{CONCLUSION}

MLT allows to achieve a high frame-rate in cardiac ultrasound imaging by transmitting multiple beams simultaneously, which unfortunately makes artifacts arise in the final image. Thanks to the application of a beamforming algorithm in RX able to lower the beam side lobes and to improve resolution at the same time, this drawback can be limited.

The paper has shown some preliminary results in simulation and phantom scans which actually demonstrate that the combined use of coherence-based beamformers and MLT could be very promising for application in cardiac imaging, achieving a similar or even better image quality than standard methods, but with higher frame-rates. Further work is foreseen to evaluate their performance in MLT echocardiography in vivo. 

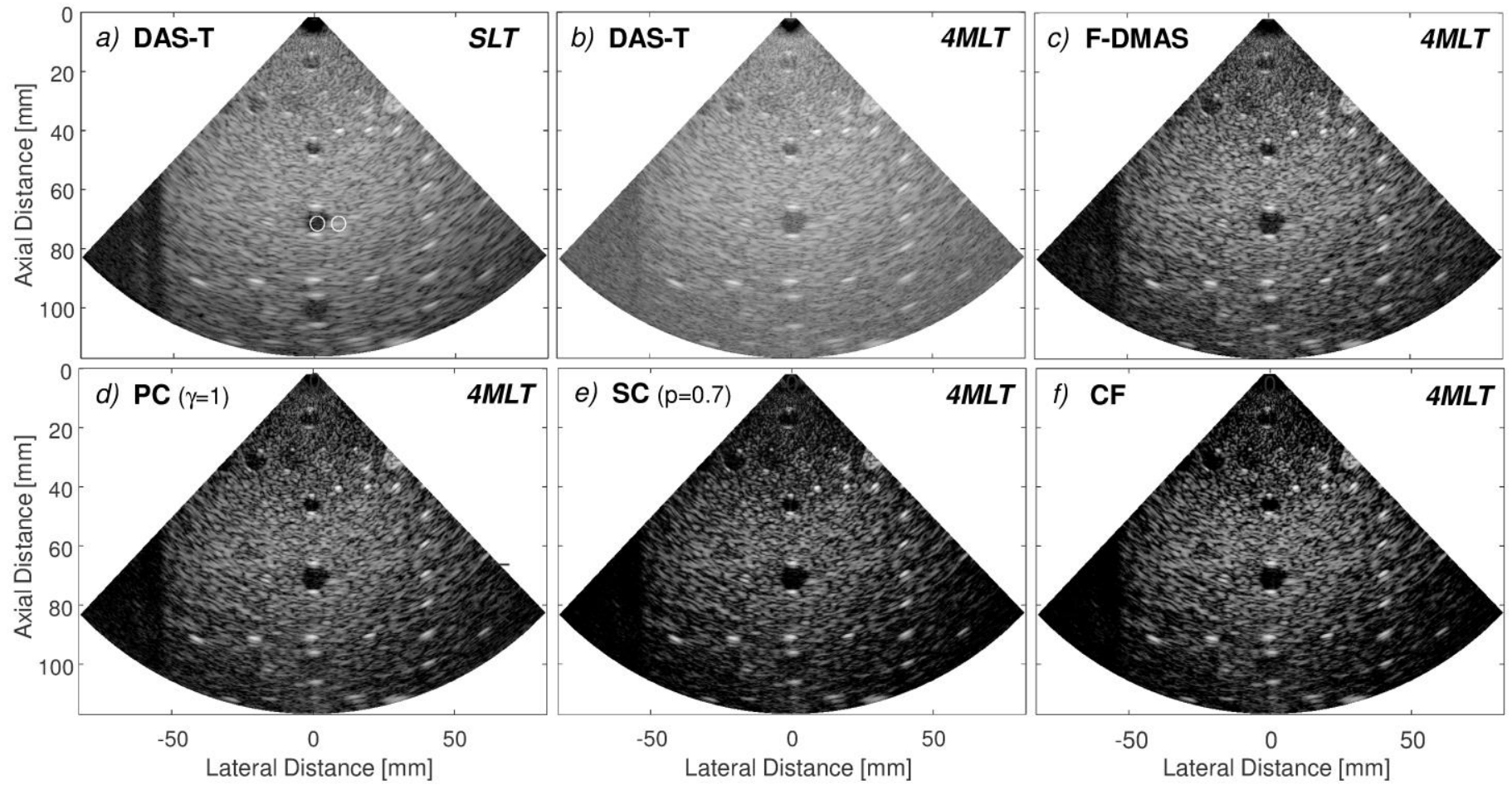

Fig. 3. Experimental phantom images obtained with Tukey TX apodization and: a), b) DAS beamforming with Tukey RX apodization (DAS-T), c) F-DMAS beamforming, d) PC with $\gamma=1, \mathrm{SC}$ with $p=0.7$, and CF weighting. Panel a) represents a standard SLT scan as a reference reference (with white circles highlighting the areas used for performance parameters computation), while panels b)-f) are obtained by implementing 4MLT. Images are represented over a $70 \mathrm{~dB}$ dynamic range (log scale).

TABle I. Performance PARAmeter VAlues

\begin{tabular}{|l|c|c|c|c|c|c|c|}
\cline { 2 - 8 } \multicolumn{1}{c|}{} & $\begin{array}{c}\text { LR } \\
{[\mathrm{mm}]}\end{array}$ & \multicolumn{2}{c|}{ CR [dB] } & \multicolumn{2}{c|}{ CNR } & \multicolumn{2}{c|}{ SSNR } \\
\cline { 2 - 8 } \multicolumn{1}{c|}{} & $\begin{array}{c}\text { SLT/ } \\
\text { 4MLT }\end{array}$ & $\boldsymbol{S L T}$ & $\mathbf{4 M L T}$ & $\boldsymbol{S L T}$ & $\mathbf{4 M L T}$ & $\boldsymbol{S L T}$ & $\mathbf{4 M L T}$ \\
\hline DAS-T & 3 & -22.3 & -15 & 1.6 & 1.4 & 1.7 & 1.7 \\
\hline F-DMAS & 2.4 & -32.5 & -22.4 & 1.4 & 1 & 1.4 & 1.1 \\
\hline PC & 1.9 & -35 & -20.9 & 1.1 & 0.9 & 1.4 & 1 \\
\hline SC & 2.1 & -37.6 & -23.6 & 1 & 0.8 & 1 & 0.8 \\
\hline CF & 2.2 & -38.6 & -26.3 & 1.2 & 0.8 & 1.2 & 0.9 \\
\hline
\end{tabular}

\section{REFERENCES}

[1] L. Tong, A. Ramalli, R. Jasaityte, P. Tortoli, J. D’hooge, "Multitransmit beam forming for fast cardiac imaging-Experimental Validation and in vivo application," IEEE. Trans. Med. Imag., vol. 33, no. 6, pp. 1205-1219, 2014

[2] A. Ramalli, A. Dallai, F. Guidi, L. Bassi, E. Boni, L. Tong, G. Fradella, J. D'hooge, and P, Tortoli, "Real-time high frame rate cardiac B-Mode and tissue Doppler imaging based on multiline transmission and multiline acquisition," IEEE. Trans. Ultrason., Ferroelectr., Freq. Control, 2018, DOI: 10.1109/TUFFC.2018.2869473.

[3] L. Tong, H. Gao, J. D'hooge, "Multi-transmit beam forming for fast cardiac imaging-A simulation study," IEEE. Trans. Ultrason., Ferroelectr., Freq. Control, vol. 60, no. 8, pp. 1719-1731, 2013.

[4] A. Rabinovich, A. Feuer, Z. Friedman, "Multi-line transmission combined with minimum variance beamforming in medical ultrasound imaging," IEEE. Trans. Ultrason. Ferroelectr. Freq. Control, vol. 62, no. 5, pp. 814-827, 2015.
[5] G. Zurakhov, L. Tong, A. Ramalli, P. Tortoli, J. D'hooge, Z. Friedman, D. Adam, "Multi line transmit beamforming combined with adaptive apodization," IEEE Trans. Ultrason. Ferroelectr. Freq. Control, vol. 65, no. 4, pp.535-545, 2018.

[6] F. Prieur, B. Dénarié, A. Austeng, H. Torp, "Multi-line transmission in medical imaging using the second-harmonic signal," IEEE Trans. Ultrason., Ferroelectr., Freq. Control, vol. 60, no. 12, pp. 2682-2692, 2013.

[7] G. Matrone, A. Ramalli, A. S. Savoia, P. Tortoli, G. Magenes, "High Frame-Rate, High Resolution Ultrasound Imaging With Multi-Line Transmission and Filtered-Delay Multiply And Sum Beamforming," IEEE Trans. Med. Imag., vol. 36, no. 2, pp. 478-486, 2017.

[8] J. Camacho, M. Parrilla, C. Fritsch, "Phase coherence imaging," IEEE Trans. Ultrason., Ferroelectr., Freq. Control, vol. 56, no. 5, pp. 958974, 2009.

[9] P. -C. $\mathrm{Li}$ and M. -L. Li, "Adaptive imaging using the generalized coherence factor," IEEE Trans. Ultrason. Ferroelectr. Freq. Control, vol. 50, no. 2, pp. 128-142, 2003.

[10] K. W. Hollmand, K. W. Rigby, and M. O'donnell, "Coherence factor of speckle from a multi-row probe," in Proc. IEEE Ultrasonics Symp., 1999, pp. 1257-1260.

[11] G. Matrone, A. S. Savoia, G. Caliano, G. Magenes, "The Delay Multiply and Sum beamforming algorithm in ultrasound B-mode medical imaging," IEEE Trans. Med. Imag., vol. 34, no. 4, pp. 940-949, 2015.

[12] J. A. Jensen and N. B. Svendsen, "Calculation of pressure fields from arbitrarily shaped, apodized, and excited ultrasound transducers," IEEE Trans. Ultrason., Ferroelectr., Freq. Control, vol. 39, no. 2, pp. 262267, 1992.

[13] J. A. Jensen, "Field: a program for simulating ultrasound systems," Med. Biol. Eng. Comput., vol. 34, pp. 351-353, 1996.

[14] E. Boni, L. Bassi, A. Dallai, F. Guidi, A. Ramalli, S. Ricci, R. J. Housden, and P. Tortoli, "A reconfigurable and programmable FPGAbased system for nonstandard ultrasound methods," IEEE Trans. Ultrason. Ferroelectr. Freq. Control, vol. 59, no. 7, pp. 1378-1385, 2012 